\title{
Assess the expression of ubiquitin specific protease USP2a for bladder cancer diagnosis
}

\author{
Pildu Jeong ${ }^{1}$, Yun-Sok Ha' ${ }^{1}$, Seok-Joong Yun ${ }^{1}$, Hyung Yoon Yoon ${ }^{1}$, Michael R. Freeman²,3, Jayoung Kim²,3*
} and Wun-Jae Kim ${ }^{1 *}$

\begin{abstract}
Background: Given that a deubiquitinating enzyme, ubiquitin-specific protease 2a (USP2a), regulates ubiquitination, trafficking, and degradation of EGFR, which plays a critical role in bladder cancer, in this study, we aimed to quantify the USP2a gene expression, and to determine the possibility that USP2a can be used for bladder cancer diagnosis.

Methods: Using two independent cohorts (cohort 1, n=339 in total; cohort 2, $n=140$ in total) consisting of human bladder tissues from BC patients and normal controls, we analyzed the gene expression levels of USP2a.

A quantitative real-time PCR amplification was performed using a Rotor Gene 6000 instrument to quantify the expression of USP2a mRNA.

Results: A comparison of 305 bladder cancers and 34 age-matched controls showed an $81.4 \%$ reduction in USP2a expression in bladder cancers as compared to normal bladder tissues $(p<0.001)$. In the independent cohort consisting of $140 \mathrm{BC}$ tissues and matched adjacent normal bladder tissues, the levels of USP2a in the specimens of BC patients were reduced by $86.9 \%$ as compared to matched surrounding normal specimens from the same patients $(p<0.001)$. Furthermore, there was $36.3 \%$ reduction of USP2a gene expression in muscle invasive bladder cancer (MIBC, $n=121$ ), compared to non muscle invasive bladder cancer (NMIBC, $n=184)(p=0.004)$. Lastly, USP2a mRNA expression was significantly reduced in higher stages of MIBC patients $(p=0.024)$, but not in NMIBC patients.
\end{abstract}

Conclusions: Our findings suggest that USP2a mRNA may be considered as a diagnostic marker candidate for bladder cancer, in particular, to stratify MIBC patients with a more invasive phenotype.

\section{Background}

Bladder cancer $(\mathrm{BC})$ is the second most common genitourinary malignancy and the fourth most common cancer among American and European [1-4]. More than $90 \%$ of bladder cancers diagnosed in the US are urothelial carcinoma, which are mostly papillary, well-, or moderately-differentiated non-muscle invasive bladder cancer (NMIBC) [5]. Within 2 years after standard treatment, approximately $20-30 \%$ of the NMIBC patients will show recurrence that results in the progression to muscle invasive disease (MIBC) [5], resulting in poor long-term survival and frequent metastases into regional lymph

\footnotetext{
* Correspondence: Jayoung.Kim@cshs.org; wjkim@chungbuk.ac.kr 2Departments of Surgery, Harvard Medical School, Boston, 02115, MA, USA 'Department of Urology, Chungbuk National University College of Medicine, Cheongju, Chungbuk, South Korea

Full list of author information is available at the end of the article
}

nodes (78 \%), liver (38 \%), lung (36 \%), bone, adrenal gland or intestine [6].

A promising finding by a research team led by François Radvanyi, along with previous studies from this group and from other laboratories [7-9], provided persuasive evidence that a certain subtype of BC-"basal-like BC"-displays activation of EGFR signaling pathway and better responsiveness to EGFR inhibitors. EGFR is known to be de-ubiqutunated by ubiquitin-specific protease 2a (USP2a) [10]. In particular, in BC cell culture system, USP2a de-ubiquitinates a cell cycle regulator, cyclin A1, thereby promoting cell proliferation [11], and USP2a regulates expression levels of fatty acid synthase (FASN), MDM2, MDM4, MDMX, and cyclin D1 [12-16]. However, USP2a has been also known to have a distinct function as a signal mediator of tumor necrosis factor- $\alpha$ (TNF- $\alpha)$-induced cell death [17], suggesting 
that USP2a function can be different in terms of cell context. Therefore, we hypothesized that USP2a expression correlates with bladder cancer progression and tested the hypothesis using our two unique cohorts consisting of patient-derived NMIBC and MIBC bladder tumor specimens.

\section{Methods}

\section{Ethics Statement}

The Ethics Committee of Chungbuk National University approved the protocol used for this study. Written informed consent was obtained from each subject. The Institutional Review Board of Chungbuk National University approved collection and analysis of all samples.

\section{Patients and Tissue Samples}

All primary tumor samples from patients who underwent TUR or radical cystectomy were histologically verified as urothelial carcinoma at Chungbuk National University in South Korea [18, 19]. Non-cancerous surrounding tissue was obtained from tissue at least $3 \mathrm{~cm}$ from the tumor and normal bladder mucosa was harvested from patients with benign diseases such as $\mathrm{BPH}$, ureter stone and stress urinary incontinence after informed consent. All control tissues were histologically confirmed as normal. Patients with concomitant carcinoma in situ, a short term follow-up period (less than 6 months), or for whom there was incomplete data collection were excluded in order to make the study population more homogeneous. The cohort 1 was consisted of 305 (253 male and 52 female with average age, 65 years old) BC patients and 34 controls (19 male and 15 female with average age, 54 years old), and the independent cohort 2 was consisted of 140 BC patients. All tumors were macro-dissected, typically within $15 \mathrm{~min}$ of surgical resection. Each bladder cancer specimen was confirmed by pathological analysis of a part of the tissue sample in fresh frozen sections from TUR or cystectomy specimens, and was then frozen in liquid nitrogen and stored at $-80{ }^{\circ} \mathrm{C}$ until use. In the case of NMIBC, a second TUR was performed 2-4 weeks after the initial resection if a bladder cancer specimen did not include the proper muscle or when a high-grade tumor was detected. Patients who had a T1 tumor, multiple tumors, large tumors $(>3 \mathrm{~cm}$ in diameter), or high grade $\mathrm{Ta}$ NMIBC received one cycle of intravesical treatment (e.g., 6-weekly treatment of BCG or 8-weekly treatment of mitomycin-C). Response to treatment was assessed by cystoscopy and urinary cytology. Patients who were free of disease within 3 months after treatment were assessed every 3 months for the first 2 years and then every 6 months thereafter. In the case of MIBC, patients with clinically localized or locally advanced tumors and good ECOG performance status (0 or 1 ) underwent radical cystectomy and complete pelvic lymph node dissection using an extended lymphadenectomy. Patients who were not eligible for radical cystectomy due to metastatic disease, poor life expectancy, or poor ECOG performance status $(\geq 2)$ underwent TUR or biopsy for histopathological diagnosis. Patients with pT3, pT4 or node-positive disease based on the analysis of radical cystectomy specimens, or with metastatic disease but good performance status, received at least four cycles of cisplatin-based chemotherapy. Patients who refused or did not complete an imaging work-up such as a CT scan or MRI at least once every 3 months to evaluate response also were excluded from analysis.

Tumors were staged and graded according to the 2002 TNM classification and the EAU guideline based on 1973 WHO grading system [20-22]. Recurrence was defined as recurrence of primary NMIBC with a lower or the same pathological stage, and progression was defined as disease with $\mathrm{T} 2$ and higher stage upon relapse in NMIBC. In case of MIBC, progression was defined as local regional recurrence or a new distant metastasis in the cystectomized group and $a \geq 20 \%$ increase in the mass of the primary tumor or a new distant metastasis in the non-cystectomized group.

\section{RNA extraction and construction of CDNA}

RNA was isolated from tissue using $1 \mathrm{ml}$ of TRIzol (Invitrogen, Carlsbad, CA) with homogenization in a 5$\mathrm{ml}$ glass tube. The homogenate was transferred to a $1.5-\mathrm{ml}$ tube and then mixed with $200 \mathrm{ml}$ of chloroform. After incubation for $5 \mathrm{~min}$ at $4{ }^{\circ} \mathrm{C}$, the homogenate was centrifuged for $13 \mathrm{~min}$ at $13,000 \mathrm{~g}$ at $4{ }^{\circ} \mathrm{C}$. The upper aqueous phase was transferred to a clean tube with $500 \mathrm{ml}$ of isopropanol. The mixture was incubated for $60 \mathrm{~min}$ at $4{ }^{\circ} \mathrm{C}$ followed by centrifugation for $8 \mathrm{~min}$ at $13,000 \mathrm{~g}, 4{ }^{\circ} \mathrm{C}$. The upper aqueous phase was discarded and mixed with $500 \mathrm{ml}$ of $75 \%$ ethanol, and centrifuged for $5 \mathrm{~min}$ at $13,000 \mathrm{~g}, 4{ }^{\circ} \mathrm{C}$. The upper aqueous layer was discarded and the pellet was dried at room temperature, dissolved in DEPC-treated water, and then stored at $-80{ }^{\circ} \mathrm{C}$. The quality and integrity of the RNA were confirmed using Nanodrop. cDNA was prepared from $1 \mathrm{mg}$ of total RNA using a First-Strand cDNA Synthesis Kit (Amersham Biosciences Europe GmbH, Freiburg, Germany) according to the manufacturer's protocol.

\section{Real-time PCR}

Real-time PCR amplification was performed using a Rotor Gene 6000 instrument (Corbett Research, Mortlake, Australia) to quantify the expression of USP2a mRNA. Real-time PCR assays were carried out in micro-reaction tubes (Corbett Research, Mortlake, Australia) using SYBR Premix EX Taq (TAKARA BIO INC., Otsu, Japan). The 
following primers were used to determine USP2a mRNA levels: forward 5' -TGCTGAGACCCGACATCACT-3'; reverse 5 '-TGGGGTCTATCCGGTAGCTA-3', as described in previous literature [12]. The PCR reaction was performed in a final volume of $10 \mathrm{ml}$ consisting of $5 \mathrm{ml}$ of 2 X SYBR premix EX Taq buffer, $0.5 \mathrm{ml}$ each of 59- and 39primer $(10 \mathrm{pmol} / \mathrm{ml})$, and $1 \mathrm{ml}$ of the sample cDNA. The product was purified with a QIAquick Extraction kit (QIAGEN, Hilden, Germany), quantified with a spectrophotometer (Perkin Elmer MBA2000, Fremont, CA), and then sequenced with an automated laser fluorescence sequencer (ABI PRISM 3100 Genetic Analyzer, Foster City, WI). Ten-fold serial dilutions of a known concentration of the product (from $100 \mathrm{pg} / \mathrm{ml}$ to $0.1 \mathrm{pg} / \mathrm{ml}$ ) were used to establish the standard curve for real-time PCR. The real-time PCR conditions were as follows: 1 cycle for $20 \mathrm{~s}$ (seconds) at $96^{\circ} \mathrm{C}$, followed by 40 cycles of $2 \mathrm{~s}$ at $96{ }^{\circ} \mathrm{C}$ for denaturation, $15 \mathrm{~s}$ at $60{ }^{\circ} \mathrm{C}$ for annealing, and $15 \mathrm{~s}$ at $72{ }^{\circ} \mathrm{C}$ for extension. The melting program was performed at $72-95^{\circ} \mathrm{C}$ with a heating rate of $1{ }^{\circ} \mathrm{C}$ per $45 \mathrm{~s}$. Spectral data were captured and analyzed using Rotor-Gene Real-Time Analysis Software 6.0 Build 14 (Corbett Research, Mortlake, Australia). All samples were run in triplicate. Glyceraldehyde-3phosphate dehydrogenase (GAPDH) was analyzed as an endogenous RNA reference gene and gene expression was normalized to the expression of GAPDH.

\section{Statistical Analysis}

Differences in continuous variables between groups were assessed by one-way ANOVA analysis.

\section{Results}

Clinical and pathological characteristics of patients with bladder cancer

A previous study from our laboratory presented evidence suggesting that the USP2a deubiquitinase plays a role in bladder cancer cell proliferation using a cell culture system [11]. In order to approach the question of whether USP2a is involved in bladder cancer progression and aggressiveness, we analyzed USP2 $a$ mRNA expression level by qRTPCR using human specimens from 305 patients with bladder cancer and 34 age-matched controls. Expression levels were compared to various clinical bladder cancer characteristics including grade $(G)$, stage $(T, N$ and $M)$, tumor size, recurrence, progression, and cancer specific survival. The characteristics of the cancer patients and controls are shown in Table 1, and further comparisons were performed in separately categorized NMIBC and MIBC groups.

\section{The level of USP2a mRNA expression as a diagnostic} marker for bladder cancer

USP $2 a$ mRNA expression in bladder cancer patients was significantly reduced $(81.4 \%)$ with respect to non-cancer
Table 1 Clinical and pathological features of patients with bladder cancer and controls

\begin{tabular}{|c|c|c|}
\hline Variables & $\begin{array}{l}\text { No. of } \\
\text { patients (\%) }\end{array}$ & $\begin{array}{l}\text { No. of } \\
\text { controls (\%) }\end{array}$ \\
\hline No. & 305 & 34 \\
\hline Mean age $\pm S D$ & $65.0 \pm 12.6$ & $53.8 \pm 14.9$ \\
\hline \multicolumn{3}{|l|}{ Gender } \\
\hline Male & $253(83.0)$ & $19(55.9)$ \\
\hline Female & $52(17.0)$ & $15(44.1)$ \\
\hline \multicolumn{3}{|l|}{ Grade } \\
\hline G1 & $56(18.4)$ & \\
\hline $\mathrm{G} 2$ & $132(43.3)$ & \\
\hline G3 & 117 (38.4) & \\
\hline \multicolumn{3}{|l|}{ T stage } \\
\hline Ta & $48(15.7)$ & \\
\hline $\mathrm{T} 1$ & $136(44.6)$ & \\
\hline $\mathrm{T} 2$ & $55(18.0)$ & \\
\hline T3 & 38 (12.5) & \\
\hline T4 & $28(9.2)$ & \\
\hline \multicolumn{3}{|l|}{ N stage } \\
\hline No & $274(89.8)$ & \\
\hline$N(1-3)$ & $31(10.2)$ & \\
\hline \multicolumn{3}{|l|}{ M stage } \\
\hline MO & $287(94.1)$ & \\
\hline M1 & $18(5.9)$ & \\
\hline
\end{tabular}

SD standard deviation

patient controls $(p<0.001)$. The reduction in MIBC $(n=121)$ when compared to NMIBC $(n=184)$ was $36.3 \%(p=0.004)$ (Table 2$)$. To further evaluate the reduction of USP2a during bladder cancer progression, we performed another qRT-PCR analysis using the second cohort, which is consisted with independent 140 patients (Table 3). BC tissues and adjacent normal bladder tissues from same patients were collected. In the cohort 2, USP2a mRNA expression of $\mathrm{BC}$ tissues was significantly decreased

Table 2 Levels of mRNA expression of USP2a were compared between bladder cancer and controls' mucosae (Cohort 1)

\begin{tabular}{llll}
\hline Variables & Patients & $\begin{array}{l}\text { mRNA expression } \\
\text { of USP2a (median with } \\
\text { IQR; } \times 103 \text { copies/ } \mu \text { l) }\end{array}$ & $p$ value \\
\hline $\begin{array}{l}\text { Cancer vs. controls' mucosae } \\
\quad \text { Controls }\end{array}$ & 34 & $825.6(370.1-1767.7)$ & $<0.001$ \\
$\quad \begin{array}{l}\text { Cancer } \\
\text { NMIBC vs. MIBC }\end{array}$ & 305 & $153.9(50.2-435.1)$ & \\
NMIBC & & & 0.004 \\
MIBC & 184 & $180.2(66.6-395.8)$ & \\
\hline
\end{tabular}

IQR interquartile range; NMIBC non-muscle invasive bladder cancer; MIBC muscle invasive bladder cancer 
Table 3 Comparisons of USP2a expression and clinopathological features of bladder cancer (Cohort 2)

\begin{tabular}{|c|c|c|c|}
\hline Variables & Patients & $\begin{array}{l}\text { mRNA expression of } \\
\text { USP2a (median with } \\
\text { IQR; } \times 103 \text { copies/ } \mu \text { l) }\end{array}$ & $P$ value \\
\hline $\begin{array}{l}\text { Cancer vs. matched } \\
\text { surrounding tissue }\end{array}$ & & & $<0.001$ \\
\hline Surrounding tissue & 140 & $1051.2(403.4-2212.3)$ & \\
\hline Cancer & 140 & $138.1(53.9-445.6)$ & \\
\hline
\end{tabular}

IQR interquartile range

in comparison to matched surrounding normal mucosae $(p<0.001)$ (Table 3). The reduction of USP2a mRNA expression in cancerous tissues was $86.9 \%$, which was similar to that seen in the first cohort in comparison to healthy controls (Table 2).

\section{USP2a mRNA expression is significantly correlated to high} stage of MIBC

With respect to several clinicopathological variables (e.g. age, sex, tumor size, number, grade, stage, and USP2a mRNA expression levels), low USP2a mRNA expression level was likely to reflect a significant risk of high stage $(p=0.024)$ in patients with MIBC, but not in NMIBC (Table 4). MIBC patients at higher stages such as T4 or $\mathrm{N}>1$ or M1 exhibited $52.5 \%$ reduced USP $2 a$ expression, compared to MIBC patients at lower stages such as T2, T3, N0 or M0 (Table 4). However, no significant alteration of USP2a level was observed according to grades (Table 4). These data suggest that USP $2 a$ expression can be used specifically as a potential marker to stratify MIBC at higher stage.

To test whether USP2a can be developed as a prognosis- or clinical outcome-related classifier, individual analyses were performed based on clinical outcomes (e.g.

Table 4 Comparisons of USP2a expression and clinopathological features of bladder cancer

\begin{tabular}{cccc}
\hline Variable & Patient (\%) & $\begin{array}{l}\text { mRNA expression of } \\
\text { USP2a (median with } \\
\text { IQR; } \times 10 \wedge 3 \text { copies/ } \mu \text { ) }\end{array}$ & p value \\
\hline Grade & & & 0.666 \\
G1 & $56(18.4)$ & $186.4(68.7-345.0)$ & \\
G2 & $132(43.3)$ & $151.4(47.3-463.2)$ & \\
G3 & $117(38.4)$ & $137.3(40.4-502.4)$ & \\
NMIBC & & & 0.681 \\
Ta & $48(26.1)$ & $160.3(68.7-340.2)$ & \\
T1 & $136(73.9)$ & $184.7(65.4-489.5)$ & \\
MIBC & & & 0.024 \\
T2 or T3, N0, M0 & $67(55.4)$ & $136.7(41.6-567.2)$ & \\
T4 or N $\geq 1$ or M1 & $54(44.6)$ & $71.7(15.8-296.1)$ & \\
\hline
\end{tabular}

$I Q R$ interquartile range; $N M I B C$ non muscle invasive bladder cancer; $M I B C$ muscle invasive bladder cancer recurrence, progression, and overall survival). Analysis was performed with 118 NMIBC patients with no recurrence vs. 66 with recurrence, 162 NMIBC patients with no progression vs. 22 with progression, 38 MIBC patients with no progression vs. 83 with progression, and 49 alive MIBC patients vs. deceased 72 subjects (Table 5). Both in NMIBC and MIBC groups, we could not detect significant differences in USP2a expression when comparing recurrence $(p=0.756$ for NMIBC), progression ( $p=0.793$ for NIBMC, and $p=0.912$ for MIBC), and overall survival ( $p=0.123$ for MIBC).

Collectively, these data suggest that (1) USP2a level may be used for stratification of cancer from normal, or NMIBC from MIBC, (2) USP2a expression is not significantly associated with recurrence, progression or overall patient survival of NMIBC as well as MIBC.

\section{Discussion}

Our results suggest that quantification of USP $2 a$ gene expression in bladder tumors may help differentiate normal vs. cancer, superficial vs. muscle invasive, and early MIBC vs. more advanced MIBC, when combined with traditional pathological testing and imaging. Analysis was conducted using 305 tissue specimens of bladder cancer patients with 34 age-matched controls, and 184 NMIBC and 121 MIBC patient tissues. A correlation between USP2a level and cancer is supported by analysis of a second cohort consisted of 140 bladder cancer specimens and their surrounding normal tissues. To our

Table 5 Comparisons of USP2a expression and clinical outcomes of bladder cancer patients

\begin{tabular}{|c|c|c|c|}
\hline Variables & Patients (\%) & $\begin{array}{l}\text { mRNA expression of } \\
\text { USP2a (median with } \\
\text { IQR; } \times 10 \wedge 3 \text { copies/ } \mu \mathrm{l})\end{array}$ & $p$ value \\
\hline \multicolumn{4}{|l|}{ NMIBC } \\
\hline Recurrence & & & 0.756 \\
\hline No recurrence & $118(64.1)$ & $200.2(77.9-391.7)$ & \\
\hline Recurrence & $66(35.9)$ & $157.6(56.4-387.2)$ & \\
\hline Progression & & & 0.793 \\
\hline No progression & $162(88.0)$ & $165.5(65.0-355.3)$ & \\
\hline Progression & $22(12.0)$ & $238.9(58.7-122.3)$ & \\
\hline \multicolumn{4}{|l|}{ MIBC } \\
\hline Progression & & & 0.912 \\
\hline No progression & $38(31.4)$ & $112.6(23.1-508.7)$ & \\
\hline Progression & $83(68.6)$ & $115.2(22.9-435.1)$ & \\
\hline Overall survival & & & 0.123 \\
\hline Alive & $49(40.5)$ & $81.3(16.1-483.3)$ & \\
\hline Death & $72(59.5)$ & $128.3(37.4-494.1)$ & \\
\hline
\end{tabular}

$I Q R$ interquartile range; NMIBC non-muscle invasive bladder cancer; $M I B C$ muscle invasive bladder cancer 
knowledge, this study presents the largest clinical data set describing USP2a gene expression in bladder cancer.

The patient-based results in this study suggest that $U S P 2 a$ expression was downregulated in bladder cancer tissues, which was inconsistent to previous in vitro studies implying the positive correlation of aggressiveness and USP $2 a[11,12]$. This discrepancies may be also explained by different and as yet poorly understood biological roles of USP2a, which is dependent on physiologic environment and cell context, or it may be the result of the natural composition of human specimens that include epithelial, stromal or inflammatory cells, and their communication with tumor cells.

There have been recent landmark studies defining the molecular phenotypes of bladder cancer, which may provide understanding the molecular and genetic events underlying $\mathrm{BC}$ progression. At least 3-4 molecular subtypes of $\mathrm{BC}$ were identified based on distinct mutations, and gene expression signatures, which may be connected to sensitivity to chemotherapy. The basal type $\mathrm{BC}$ subgroup expressing basal markers was dependent on the EGFR signaling pathway, thus this subgroup was significantly sensitive to treatment with drugs that inhibit the EGFR pathway. EGFR, a potential prognostic marker for MIBC has also been widely studied in bladder cancer [18]. Up-regulated EGFR signaling is known to initiate a cascade of events and lead to cell proliferation, migration, invasion and inhibition of apoptosis, all of which promotes tumor progression [23]. Altered expression of EGF family members (e.g. EGF, epiregulin and HB-EGF (heparin-binding epidermal growth factor-like growth factor)) and EGFR has been suggested as mediators of bladder cancer progression [24]. We showed previously that HB-EGF accumulates in the nucleus in aggressive TCC cells and is involved in an EGFR-dependent autocrine loop [24, 25], and that USP2a increases EGFR stability by inhibiting endocytosis and degradation of EGFR [10]. Based on the published literatures and the present data, EGFR and USP $2 a$ levels may be correlated with disease progression, although this has not been evaluated in the same series of tumors. A next step is to determine whether altered levels of EGFR [18] and USP2 $a$ are correlated with more aggressive bladder cancer, and whether these combined two molecular markers might predict aggressive disease.

Collectively, there is a critical need for methods that identify patients with MIBC that are likely to experience disease progression or metastasis. Our study demonstrated that USP2a expression levels make it possible to distinguish bladder cancer from normal, and muscle-invasive from non-invasive disease.

\section{Conclusions}

The experimental results in this study provide an evidence suggesting that USP2a as a biomarker may improve diagnosis of human bladder cancer patients. Although the present analyses were performed with a large series of bladder cancer patients, which were carefully grouped into NMIBC vs. MIBC based on clinical evaluation, a larger prospective validation of our findings should be attempted.

\section{Abbreviations}

NMIBC: Non-muscle invasive bladder cancer; MIBC: Muscle invasive bladder cancer; EGFR: Epidermal growth factor receptor; DUBs: Deubiquitinating enzymes; USP2a: Ubiquitin-specific protease 2a; FASN: Fatty acid synthase; OSCC: Oral squamous cell carcinoma; AJCC: American Joint Committee on Cancer; UHRF1: E3 ubiquitin-protein ligase UHRF1; PFKB: 6-phosphofructo-2kinase/fructose-2,6-biphosphatase; KPNA2: Karyopherin-a2; GSTF6: Glutathione S-transferase 1; S100A8: S100 calcium binding protein A8; FGFR3: Fibroblast growth factor receptor-3; IL-1B: Interleukin-1 $\beta$; TUR: Transurethral resection.

\section{Competing interests}

The authors declare that they have no competing interests.

\section{Authors' contributions}

PJ and YH performed experiments, and participated data interpretation. SY and $\mathrm{HY}$ participated in the design of the study and performed the statistical analysis. JK and WK supervised study design and experiments, and led obtaining funding. SY and JK drafted the manuscript. MF participated critical revision of the manuscript. All authors read and approved the final manuscript.

\section{Acknowledgements}

This research was supported by Basic Science Research Program through the National Research Foundation of Korea (NRF) funded by the Ministry of Science, ICT \& Future Planning (No. 2014R1A2A1A09006983,

2015R1A2A2A03004100 and 2013R1A1A2004740); and by NIH grants 1R01DK100974-01, U24 DK097154, NIH NCATS UCLA CTSI UL1TR000124, the Steven Spielberg Discovery Fund in Prostate Cancer Research Career Development Award, Interstitial Cystitis Association (ICA) Imagine NO IC Pilot Grant, and a Fishbein Family Research Grant (to J.K.); J.K. is an American Urological Association Foundation Research Scholar and the Eleanor and Miles Shore Scholar at Harvard Medical School.

\section{Author details}

${ }^{1}$ Department of Urology, Chungbuk National University College of Medicine, Cheongju, Chungbuk, South Korea. ${ }^{2}$ Departments of Surgery, Harvard Medical School, Boston, 02115, MA, USA. ${ }^{3}$ Cancer Biology Division, Departments of Surgery and Biomedical Sciences, Cedars-Sinai Medical Center, Los Angeles, CA 90048, USA.

Received: 24 February 2015 Accepted: 27 July 2015

Published online: 07 August 2015

\section{References}

1. Kirkali Z, Chan T, Manoharan M, Algaba F, Busch C, Cheng L, et al. Bladder cancer: epidemiology, staging and grading, and diagnosis. Urology. 2005;66(6 Suppl 1):4-34.

2. Noon AP, Catto JW. Bladder cancer in 2012: challenging current paradigms. Nat Rev Urol. 2013;10(2):67-8.

3. Resnick MJ, Bassett JC, Clark PE. Management of superficial and muscle-invasive urothelial cancers of the bladder. Curr Opin Oncol. 2013;25(3):281-8.

4. Richter S, Sridhar SS. New directions for biologic targets in urothelial carcinoma. Mol Cancer Ther. 2012;11(6):1226-35.

5. Babjuk M, Oosterlinck W, Sylvester R, Kaasinen E, Bohle A, Palou-Redorta J. EAU guidelines on non-muscle-invasive urothelial carcinoma of the bladder. Eur Urol. 2008;54(2):303-14.

6. Stenzl A, Cowan NC, De Santis M, Kuczyk MA, Merseburger AS, Ribal MJ, et al. Treatment of muscle-invasive and metastatic bladder cancer: update of the EAU guidelines. Eur Urol. 2011;59(6):1009-18.

7. Rebouissou S, Bernard-Pierrot I, de Reynies A, Lepage ML, Krucker C, Chapeaublanc E, et al. EGFR as a potential therapeutic target for a subset of muscle-invasive bladder cancers presenting a basal-like phenotype. Sci Transl Med. 2014;6(244):244ra291. 
8. Cancer Genome Atlas Research N. Comprehensive molecular characterization of urothelial bladder carcinoma. Nature. 2014;507(7492):315-22

9. Choi W, Porten S, Kim S, Willis D, Plimack ER, Hoffman-Censits J, et al. Identification of distinct basal and luminal subtypes of muscle-invasive bladder cancer with different sensitivities to frontline chemotherapy. Cancer Cell. 2014;25(2):152-65.

10. Liu Z, Zanata SM, Kim J, Peterson MA, Di Vizio D, Chirieac LR, et al. The ubiquitin-specific protease USP2a prevents endocytosis-mediated EGFR degradation. Oncogene. 2012;32(13):1660-9.

11. Kim J, Kim WJ, Liu Z, Loda M, Freeman MR. The ubiquitin-specific protease USP2a enhances tumor progression by targeting cyclin A1 in bladder cancer. Cell Cycle. 2012:11(6):1123-30.

12. Priolo C, Tang D, Brahamandan M, Benassi B, Sicinska E, Ogino S, et al. The isopeptidase USP2a protects human prostate cancer from apoptosis. Cancer Res. 2006;66(17):8625-32.

13. Stevenson LF, Sparks A, Allende-Vega N, Xirodimas DP, Lane DP, Saville MK The deubiquitinating enzyme USP2a regulates the p53 pathway by targeting Mdm2. EMBO J. 2007;26(4):976-86.

14. Allende-Vega N, Sparks A, Lane DP, Saville MK. MdmX is a substrate for the deubiquitinating enzyme USP2a. Oncogene. 2010;29(3):432-41.

15. Shi $Y$, Solomon LR, Pereda-Lopez A, Giranda $V L$, Luo $Y$, Johnson EF, et al. The deubiquitinase USP2A regulates the stability of aurora-A. J Biol Chem. 2011;286(45):38960-8.

16. da Silva SD, Cunha IW, Nishimoto IN, Soares FA, Carraro DM, Kowalski LP, et al. Clinicopathological significance of ubiquitin-specific protease 2a (USP2a), fatty acid synthase (FASN), and ErbB2 expression in oral squamous cell carcinomas. Oral Oncol. 2009:45(10):e134-139.

17. Mahul-Mellier AL, Pazarentzos E, Datler C, Iwasawa R, Abuali G, Lin B, Grimm S. De-ubiquitinating protease USP2a targets RIP1 and TRAF2 to mediate cell death by TNF. Cell Death Differ. 2011; doi: 10.1038/cdd.2011.185

18. Kim WJ, Kim SK, Jeong P, Yun SJ, Cho IC, Kim IY, et al. A four-gene signature predicts disease progression in muscle invasive bladder cancer. Mol Med. 2011;17(5-6):478-85

19. Kim WJ, Kim EJ, Kim SK, Kim YJ, Ha YS, Jeong P, et al. Predictive value of progression-related gene classifier in primary non-muscle invasive bladder cancer. Mol Cancer. 2010:9:3.

20. Epstein JI, Amin MB, Reuter VR, Mostofi FK. The World Health Organization/ International Society of Urological Pathology consensus classification of urothelial (transitional cell) neoplasms of the urinary bladder. Bladder Consensus Conference Committee. Am J Surg Pathol. 1998;22(12):1435-48.

21. Babjuk M, Oosterlinck W, Sylvester R, Kaasinen E, Bohle A, Palou-Redorta J, et al. EAU guidelines on non-muscle-invasive urothelial carcinoma of the bladder, the 2011 update. Eur Urol. 2011;59(6):997-1008.

22. Schned AR, Andrew AS, Marsit CJ, Zens MS, Kelsey KT, Karagas MR. Survival following the diagnosis of noninvasive bladder cancer: WHO/International Society of Urological Pathology versus WHO classification systems. J Urol. 2007:178(4 Pt 1):1196-200. discussion 1200.

23. Dangle PP, Zaharieva B, Jia H, Pohar KS. Ras-MAPK pathway as a therapeutic target in cancer-emphasis on bladder cancer. Recent Pat Anticancer Drug Discov. 2009:4(2):125-36.

24. Adam RM, Danciu T, McLellan DL, Borer JG, Lin J, Zurakowski D, et al. A nuclear form of the heparin-binding epidermal growth factor-like growth factor precursor is a feature of aggressive transitional cell carcinoma. Cancer Res. 2003:63(2):484-90.

25. Kim J, Adam RM, Freeman MR. Trafficking of nuclear heparin-binding epidermal growth factor-like growth factor into an epidermal growth factor receptor-dependent autocrine loop in response to oxidative stress Cancer Res. 2005:65(18):8242-9.

\section{Submit your next manuscript to BioMed Central and take full advantage of:}

- Convenient online submission

- Thorough peer review

- No space constraints or color figure charges

- Immediate publication on acceptance

- Inclusion in PubMed, CAS, Scopus and Google Scholar

- Research which is freely available for redistribution

Submit your manuscript at www.biomedcentral.com/submit 